\title{
PARALLEL AND SERIAL JUMPS OF WEAK WEAK KÖNIG'S LEMMA
}

\author{
LAURENT BIENVENU AND RUTGER KUYPER
}

\begin{abstract}
We study the principle of positive choice in the Weihrauch degrees In particular, we study its behaviour under composition and jumps, and answer three questions asked by Brattka, Gherardi and Hölzl.
\end{abstract}

\section{INTRODUCTION}

In this paper we study the computational strength of positive choice for the spaces $\mathcal{X} \in\left\{2^{\omega}, \omega \times 2^{\omega}, \omega^{\omega}\right\}$. Here, positive choice is the principle which assigns to a tree of positive measure the collection of paths through that tree; a different name for $\mathrm{PC}_{2}$ is weak weak König's lemma or WWKL. There are several different approaches to classifying the relative strength of different principles; for example, one could study the relative strength over a weak base system such as $\mathrm{RCA}_{0}$, as is commonly done in reverse mathematics. However, in this paper we study these principles in the Weihrauch degrees, which imposes several restrictions when we are comparing two principles $\Phi$ and $\Psi$ : for $\Phi$ to Weihrauch-reduce to $\Psi$, which intuitively means that $\Phi$ is 'easier' than $\Psi$, we should be able to solve $\Phi$ using one instance of $\Psi$ in a uniform way.

In particular, in the Weihrauch degrees it makes sense to ask whether applying a principle twice in a row is strictly stronger than only using it once. In fact, given two principles $\Phi$ and $\Psi$ there is a natural degree $\Phi \star \Psi$ corresponding to applying $\Phi$ after $\Psi$, as shown by Brattka and Pauly [6]; we call $\Phi \star \Psi$ the compositional product of $\Phi$ and $\Psi$.

One natural way of strengthening a principle $\Phi$ is by weakening the representation of its input. For example, when talking about positive choice, instead of considering the principle which takes as input a tree of positive measure and outputs a path through the tree, we could consider the principle which takes as input a sequence of trees which converges pointwise to a tree of positive measure; i.e., the input is only a $\Delta_{2}^{0}$-representation of the intended tree. This can be done in general, and so, for every principle $\Phi$ there is a principle $\Phi^{\prime}$, the jump of $\Phi$.

It is now natural to study the interaction of these different operations. For example, Brattka, Gherardi and Marcone [4] studied the jump of weak König's lemma (the principle which assigns to an infinite binary tree the set of paths through that tree), and showed that

$$
\mathrm{WKL}^{\prime} \star \mathrm{WKL}^{\prime} \equiv_{W} \mathrm{WKL}^{\prime \prime}
$$

Brattka, Gherardi and Hölzl [3] studied various properties of probabilistic choice; for example, they showed that

$$
\mathrm{PC}_{\mathcal{X}} \star \mathrm{PC}_{\mathcal{X}} \equiv_{W} \mathrm{PC}_{\mathcal{X}}
$$

They concluded their paper with several questions. First of all, they asked:

Date: January 31, 2017.

The research of the second author was supported by John Templeton Foundation grant 15619: 'Mind, Mechanism and Mathematics: Turing Centenary Research Project'. 
Is WWKL' closed under composition?

Given their result, this would be a natural relativisation 'one jump up'.

On the other hand, by the result from [4], iterating $\mathrm{WKL}^{\prime}$ brings us up to WKL". So, another natural question is:

$$
\text { Or is } \mathrm{WWKL}^{\prime} \star \mathrm{WWKL}^{\prime} \equiv_{W} \mathrm{WWKL}^{\prime \prime} \text { ? }
$$

We will show that neither of these is the case. In fact, we will show in section 4 that

$$
\mathrm{WWKL}^{\prime} \star \mathrm{WWKL}^{\prime} \equiv_{W} \mathrm{PC}_{\omega \times 2^{\omega}}^{\prime},
$$

and relativising a result from Brattka and Pauly [7] we show that

$$
\mathrm{WWKL}^{\prime}<_{W} \mathrm{PC}_{\omega \times 2^{\omega}}^{\prime}<_{W} \mathrm{WWKL}^{\prime \prime} .
$$

The third question asked in [3] is

$$
\text { Is } \mathrm{WWKL}^{\prime} \leq_{W} \mathrm{PC}_{\omega} \omega \text { ? }
$$

We also give a negative answer to this question. In fact, we show in section 5 that both $\mathrm{PC}_{\omega \times 2} \equiv_{s W} \quad \mathrm{PC}_{\omega^{\omega}}$ and $\mathrm{PC}_{\omega \times 2^{\omega}}^{\prime} \equiv_{s W} \quad \mathrm{PC}_{\omega^{\omega}}^{\prime}$, which we combine with the theorem from [7] that $\mathrm{PC}_{\omega \times 2}{ }^{\omega}<_{W}$ WWKL'.

Finally, in section 6 we study the remaining compositions $f \star g$ for $f, g \in$ $\left\{\mathrm{WWKL}, \mathrm{WWKL}, \mathrm{PC}_{\omega \times 2^{\omega}}, \mathrm{PC}_{\omega \times 2^{\omega}}^{\prime}\right\}$. The results are summarised in section 7 .

We assume that the reader is familiar with basic notions of computability theory and algorithmic randomness, and refer to $[8,9]$ for a good treatment of both subjects.

Our notation is mostly standard. We use $f: \subseteq \mathcal{X} \rightarrow \mathcal{Y}$ to denote that $f$ is a partial map, and we use $f: \mathcal{X} \rightrightarrows \mathcal{Y}$ to denote that $f$ is a multi-valued function. Whenever we talk about a path through a tree, we mean an infinite path. When $T^{\emptyset^{\prime}}$ is a $\Delta_{2}^{0}$ tree, we denote by $T[s]$ the set of strings $\sigma$ such that for no string $\tau \subseteq \sigma$ we have that $T^{\emptyset^{\prime}[s]}(\tau)[s] \downarrow=0$, i.e., we make sure $T[s]$ is also a tree. We fix once and for all a computable bijection $\langle.,$.$\rangle between \omega^{2}$ and $\omega$. If $f: \omega \rightarrow \mathcal{Y}$ is a function, $f^{[i]}$ is the function defined by $f^{[i]}(n)=f(\langle i, n\rangle)$.

\section{WeIHRAUCH DEGREES}

In this section we will repeat the necessary definitions and background on Weihrauch reducibility. The definition of Weihrauch reducibility has gone through several generalisations, culminating in the current definition of Brattka and Gherardi [2]. This is the definition we give here.

As is known in computability theory, Baire space $\omega^{\omega}$ can be used to represent many different kind of things, from trees to real numbers. In order to properly define Weihrauch reducibility, we need to make these representations explicit. We do this through the notion of a represented space.

Definition 2.1. A representation of a set $\mathcal{X}$ is a surjective partial map $\delta: \subseteq \omega^{\omega} \rightarrow$ $\mathcal{X}$. We say that $\left(\mathcal{X}, \delta_{\mathcal{X}}\right)$ is a represented space.

Now, we consider multi-valued (partial) functions $f: \subseteq \mathcal{X} \rightrightarrows \mathcal{Y}$, i.e., partial maps which send an $x \in \operatorname{dom}(f)$ to a non-empty subset of $Y$. Henceforth, we will omit the word 'partial' and talk about multi-valued partial functions just as multi-valued functions. An easy example of a multi-valued function is the following, which will be relevant throughout this paper.

Definition 2.2. For $n$ a positive integer, let $n-\operatorname{Ran}: 2^{\omega} \rightrightarrows 2^{\omega}$ be the multi-valued function which sends $X$ to the set of $n$-random reals relative to $X$, i.e., those reals which are Martin-Löf random relative to $X^{(n-1)}$. 
To any multi-valued function $f: \subseteq \mathcal{X} \rightrightarrows \mathcal{Y}$ we can assign a set of partial functions from $\mathcal{X}$ to $\mathcal{Y}$ in a natural way: take the set of choice functions, i.e., the set of functions $F: \subseteq \mathcal{X} \rightarrow \mathcal{Y}$ such that $\operatorname{dom}(F)=\operatorname{dom}(f)$, and $F(x) \in f(x)$ for every $x \in \operatorname{dom}(f)$.

Furthermore, given any multi-valued function $f: \subseteq \mathcal{X} \rightrightarrows \mathcal{Y}$ and representations $\delta_{\mathcal{X}}$ of $\mathcal{X}$ and $\delta_{\mathcal{Y}}$ of $\mathcal{Y}$, we can represent the function $f$ as a function $g: \subseteq \omega^{\omega} \rightrightarrows \omega^{\omega}$, although not necessarily uniquely if $\delta \mathcal{y}$ is not injective. For example, looking at $n$-Ran, if we let $\delta_{2} \omega$ be the inclusion of $2^{\omega}$ in $\omega^{\omega}$, we can 'pull back' this function to $\omega^{\omega}$ by letting $g$ be the function sending $x \in 2^{\omega}$ to $f(x)$, and being undefined outside of $2^{\omega}$.

In what follows, we do not just think of a multi-valued function $f: \subseteq \mathcal{X} \rightrightarrows \mathcal{Y}$ as a set-theoretic multi-valued function, but we actually think of it as a multi-valued function $f: \subseteq\left(X, \delta_{X}\right) \rightrightarrows\left(Y, \delta_{Y}\right)$ from a represented space to a represented space. In other words, we are thinking of an explicit representation of the domain and codomain. However, when the representations are clear and there is no possible confusion we will often write $f: \subseteq X \rightrightarrows Y$ anyway.

Combining these ideas, we are lead to the notion of a realiser.

Definition 2.3. Let $f: \subseteq\left(\mathcal{X}, \delta_{\mathcal{X}}\right) \rightrightarrows\left(\mathcal{Y}, \delta_{\mathcal{Y}}\right)$ be a multi-valued function. We say that $F: \subseteq \omega^{\omega} \rightarrow \omega^{\omega}$ is a realiser of $f$, written as $F \vdash f$, if for every $z \in \operatorname{dom}(f \circ \delta \mathcal{X})$ we have $z \in \operatorname{dom}(F)$, and $\delta_{\mathcal{Y}}(F(z)) \in f\left(\delta_{\mathcal{X}}(z)\right)$.

As an example, a realiser of $n$-Ran is now just a function which assigns to every $X \in 2^{\omega}$ an $n$-random real relative to $X$.

The notion of Weihrauch reducibility now defines what it means for a multivalued function $f: \subseteq \mathcal{X} \rightrightarrows \mathcal{Y}$ to be 'easier' than a multi-valued function $g: \subseteq \mathcal{U} \rightrightarrows \mathcal{V}$, in the sense that the realisers of $g$ uniformly compute realisers of $f$. This is made precise in the definition below.

Definition 2.4. Let $f, g$ be multi-valued functions (on represented spaces). Then we say that $f$ is Weihrauch reducible to $g$, written as $f \leq_{W} g$, if there exist Turing functionals $K: \subseteq \omega^{\omega} \rightarrow \omega^{\omega}$ and $H: \subseteq \omega^{\omega} \times \omega^{\omega} \rightarrow \omega^{\omega}$ such that for every $G$ with $G \vdash g$ we have that $H($ id, $G \circ K) \vdash f$.

Furthermore, we say that $f$ is strongly Weihrauch reducible to $g$, written as $f \leq_{s W} g$, if there exist Turing functionals $K, H: \subseteq \omega^{\omega} \rightarrow \omega^{\omega}$ such that for every $G$ with $G \vdash g$ we have that $H(G \circ K) \vdash f$.

If $f \leq_{W} g$ and $K$ and $H$ are as in the definition above, we say that $K$ and $H$ witness that $f \leq_{W} g$.

The difference between regular Weihrauch reducibility and strong Weihrauch reducibility is that in the first case the post-processor $H$ has access to the original input, while this is lost in the latter case.

As for other reducibilities in computability theory, this induces a degree structure in the usual way. That is, we say that $f$ is Weihrauch equivalent to $g$, or $f \equiv_{W} g$, if both $f \leq_{W} g$ and $g \leq_{W} f$. We say that the equivalence class of $f$ under $\equiv_{W}$ is the Weihrauch degree of $f$. We can introduce strong Weihrauch equivalence $\equiv_{s W}$ and the strong Weihrauch degrees in the same way.

In [4], Brattka, Gherardi and Marcone have introduced a natural operation related to composition, as mentioned in the introduction. This notion is called the compositional product.

Definition 2.5. Let $f: \subseteq \mathcal{X} \rightrightarrows \mathcal{Y}$ and $g: \subseteq \mathcal{Y} \rightrightarrows \mathcal{Z}$. Then $g \circ f: \subseteq \mathcal{X} \rightrightarrows \mathcal{Z}$ is the multi-valued function with domain

$$
\{x \in \mathcal{X} \mid x \in \operatorname{dom}(f) \wedge f(x) \subseteq \operatorname{dom}(g)\},
$$


and for $x$ in the domain of $g \circ f$ we have

$$
g \circ f(x)=\{z \in \mathcal{Z} \mid \exists y \in \mathcal{Y}(z \in g(y) \wedge y \in f(x))\} .
$$

Definition 2.6. Let $f, g$ be multi-valued functions (on represented spaces). Then

$$
f \star g=\max \left(f_{0} \circ g_{0} \mid f_{0} \leq_{W} f \text { and } g_{0} \leq_{W} g\right),
$$

where the maximum is taken over those $f_{0}$ and $g_{0}$ where the codomain of $g_{0}$ and the domain of $f_{0}$ coincide.

That the supremum exists and that it is even a maximum was proven in [6]. Let us give an example using randomness to illustrate how to work with these compositional products and how to formally work with Weihrauch reducibility.

\section{Proposition 2.7.}

$$
n-\operatorname{Ran} \star n-\operatorname{Ran} \equiv_{W} n-\operatorname{Ran} .
$$

Proof. It is not hard to see that $f \leq_{W} f \star f$ always holds: consider the composition of $f$ and the identity.

Conversely, let $f_{0}: \subseteq\left(X, \delta_{X}\right) \rightrightarrows\left(Y, \delta_{Y}\right)$ and $g_{0}: \subseteq\left(Z, \delta_{Z}\right) \rightrightarrows\left(X, \delta_{X}\right)$ with $f_{0}, g_{0} \leq_{W} n$-Ran, and let this be witnessed by $V_{0}$ and $U_{0}$ for $f_{0}$, and by $K_{0}$ and $H_{0}$ for $g_{0}$. We now define $K$ to be the identity, and we let $H$ be the function sending $(x, y \oplus z) \in \omega^{\omega} \times \omega^{\omega}$ to $U_{0}\left(H_{0}(x, y), z\right)$. We claim: $K$ and $H$ witness that $f_{0} \circ g_{0} \leq_{W} n-\operatorname{Ran}$.

Thus, let $G \vdash n$-Ran; we need to show that $H($ id, $G \circ K) \vdash f_{0} \circ g_{0}$. So, let $x \in \omega^{\omega}$ be in the domain of $f_{0} \circ g_{0} \circ \delta_{Z}$. Then

$$
H(x, G(K(x)))=U_{0}\left(H_{0}\left(x, G_{0}(x)\right), G_{1}(x)\right),
$$

where $G(x)=G_{0}(x) \oplus G_{1}(x)$. Note that $G_{0}(x)$ is $n$-random relative to $x$, hence it is also $n$-random relative to $K_{0}(x)$. Thus, per choice of $K_{0}$ and $H_{0}$ we see that $\delta_{X}\left(H_{0}\left(x, G_{0}(x)\right)\right) \in g_{0}\left(\delta_{Z}(x)\right)$. Next, by van Lambalgen's theorem for $n$ randomness (see e.g. [8, Corollary 6.9.3]) we know that $G_{1}(x)$ is $n$-random relative to $x \oplus G_{0}(x)$, so it is also $n$-random relative to $V_{0}\left(H_{0}\left(x, G_{0}(x)\right)\right)$. Therefore

$$
\delta_{Y}\left(U_{0}\left(H_{0}\left(x, G_{0}(x)\right), G_{1}(x)\right)\right) \in f_{0}\left(\delta_{X}\left(H_{0}\left(x, G_{0}(x)\right)\right)\right) \subseteq\left(f_{0} \circ g_{0}\right)\left(\delta_{Z}(x)\right),
$$

as desired.

\section{Probabilistic Choice}

In Brattka, Gherardi and Hölzl [3], various choice principles are studied within the Weihrauch degrees. The main focus of this paper will be probabilistic choice, for which we will recall the definition shortly. While in [3] various spaces are studied, we will only study Cantor space $2^{\omega}$, Baire space $\omega^{\omega}$ and the intermediate space $\omega \times 2^{\omega}$, which allows us to simplify the necessary definitions.

Definition 3.1. We let Tree $2^{\omega}$ be the set of trees in $2^{<\omega}$, we let $\operatorname{Tree}_{\omega^{\omega}}$ be the set of trees in $\omega^{<\omega}$ and we let Tree $\omega \times 2^{\omega}$ be the set of trees in $\{\emptyset\} \cup\left(\omega \times 2^{<\omega}\right)$, where a tree in $Y$ is a subset of $Y$ closed under taking substrings. For any tree $T$, we let $[T]$ be the set of infinite paths through $T$.

In what follows, we will assume reasonable fixed representations of Tree $2^{\omega}$, Tree $\omega^{\omega}$ and Tree $\omega \times 2^{\omega}$, where 'reasonable' means that membership of a string $\sigma$ in a tree $\delta(X)$ should be uniformly decidable in $X$.

There are natural Borel measures on the three spaces mentioned above: on Cantor space, we have the measure $\mu_{2}$ induced by

$$
\mu_{2 \omega}(\llbracket \sigma \rrbracket)=2^{-|\sigma|}
$$


(where $\llbracket \sigma \rrbracket$ is the set of $x \in 2^{\omega}$ extending $\sigma$ ). This corresponds to the probability measure where each bit has value 0 or 1 , each with probability $1 / 2$, independently of other bits. On Baire space we have the measure $\mu_{\omega^{\omega}}$ induced by

$$
\mu_{\omega^{\omega}}(\llbracket \sigma \rrbracket)=\prod_{i<|\sigma|} 2^{-\sigma(i)-1}
$$

that is, each value of the sequence is equal to $n$ with probability $2^{-n-1}$, independently of all other values. On $\omega \times 2^{\omega}$ we have the measure induced by

$$
\mu_{\omega \times 2^{\omega}}(\llbracket \sigma \rrbracket)=2^{-\sigma(0)-1} 2^{-|\sigma|+1},
$$

that is, the first value equal to $n$ with probability $2^{-n-1}$ and every other value is 0 or 1 with probability $1 / 2$, all values being independent.

Given any tree, we call 'measure of $T$ ' the measure of $[T]$. Clearly, every tree of positive measure has an infinite path. Probabilistic choice is the multi-valued function assigning to such a tree of positive measure the collection of its paths.

Definition 3.2. Given $\mathcal{X} \in\left\{2^{\omega}, \omega^{\omega}, \omega \times 2^{\omega}\right\}$, we let Tree ${ }_{\mathcal{X}}^{>0} \subseteq$ Tree $_{\mathcal{X}}$ be the set of trees of positive measure.

Definition 3.3. Given $\mathcal{X} \in\left\{2^{\omega}, \omega^{\omega}, \omega \times 2^{\omega}\right\}$, we let $\mathrm{PC}_{\mathcal{X}}: \operatorname{Tree}_{\mathcal{X}}^{>0} \rightrightarrows \mathcal{X}$ be the multi-valued function sending a tree $T$ of positive measure to $[T]$. Alternatively, we call $\mathrm{PC}_{2}$ weak weak König's lemma, or WWKL.

As a warmup, let us compare randomness and PC.

Proposition 3.4. We have $1-\operatorname{Ran} \leq_{s W} \mathrm{PC}_{2^{\omega}}$ but $\mathrm{PC}_{2^{\omega}} \underline{L}_{W} 1-\mathrm{Ran}$.

Proof. Fix a universal oracle Martin-Löf test $\mathcal{U}_{0}^{X}, \mathcal{U}_{1}^{X}, \ldots$ and let $T^{X}$ be a tree uniformly computable in $X$ such that $\left[T^{X}\right]$ is the complement of $\mathcal{U}_{0}^{X}$. Now let $K$ be the total Turing functional sending $X$ to $T^{X}$, and let $H$ be the identity. Then $K$ and $H$ witness that $1-\operatorname{Ran} \leq_{s W} \mathrm{PC}_{2} \omega$.

For the converse, see Brattka, Hendtlass and Kreuzer [5].

As informally explained in the introduction, there is a notion of a jump in the Weihrauch degrees, introduced in Brattka, Gherardi and Marcone [4].

Definition 3.5. Given any multi-valued function $f: \subseteq\left(X, \delta_{X}\right) \rightrightarrows\left(Y, \delta_{Y}\right)$, we obtain its jump $f^{\prime}$ by replacing the representation $\delta_{X}$ by $\delta_{X}^{\prime}=\delta_{X}$ olim, where $\lim : \subseteq$ $\omega^{\omega} \rightarrow \omega^{\omega}$ is the partial function sending $f$ to the pointwise limit of $f^{[0]}, f^{[1]}, \ldots$, where the domain of lim is exactly the set of $f$ for which this limit exists.

In other words, as a set-theoretic function $f^{\prime}$ is the same as $f$, but its input representation is weakened by only giving a sequence converging to some $z$, instead of the actual intended input $z$. In the case of PC, this leads to the following.

Definition 3.6. Let $\mathcal{X} \in\left\{2^{\omega}, \omega^{\omega}, \omega \times 2^{\omega}\right\}$. We let limtree ${ }_{\mathcal{X}}^{>0}$ be the collection of sequences $\left(T_{i}\right)_{i \in \omega}$ with $T_{i} \in \operatorname{Tree}_{\mathcal{X}}$ such that $\left(T_{i}\right)_{i \in \omega}$ converges pointwise to a tree $T_{\infty}$ of positive measure.

Again, we have a natural representation of limtree $\mathcal{X}_{\mathcal{X}}^{>0}$ by sending $f \in \omega^{\omega}$ to $\left(\delta_{\operatorname{Tree}_{\mathcal{X}}}\left(f^{[i]}\right)\right)_{i \in \omega}$.

Proposition 3.7. Let $\mathcal{X} \in\left\{2^{\omega}, \omega^{\omega}, \omega \times 2^{\omega}\right\}$. Given any multi-valued function $f: \subseteq$ Tree $_{\mathcal{X}}^{>0} \rightrightarrows Y$, let $\phi(f): \subseteq$ limtree ${ }_{\mathcal{X}}^{>0} \rightrightarrows Y$ be the multi-valued function sending $\left(T_{i}\right)_{i \in \omega}$ to $f\left(T_{\infty}\right)$. Then $\phi$ is a bijection between $\left\{f \mid f: \subseteq \operatorname{Tree}_{\mathcal{X}}^{>0} \rightrightarrows Y\right\}$ and $\left\{g \mid g: \subseteq\right.$ limtree $\left._{\mathcal{X}}^{>0} \rightrightarrows Y\right\}$. Furthermore, $f^{\prime}$ and $\phi(f)$ have exactly the same realisers. 
Proof. The inverse of $\phi$ is the function sending $g: \subseteq \operatorname{limtree}_{\mathcal{X}}^{>0} \rightrightarrows Y$ to the multivalued function sending a tree $T$ to $g\left((T)_{i \in \omega}\right)$, where $(T)_{i \in \omega}$ is the sequence that is constantly $T$. That $f^{\prime}$ and $\phi(f)$ have the same realisers follows directly from unfolding the definitions.

Thus, in particular we can identify $\mathrm{PC}_{\mathcal{X}}^{\prime}$ with the multi-valued function sending an element $\left(T_{i}\right)_{i \in \omega} \in$ limtree $_{\mathcal{X}}^{>0}$ to $\left[T_{\infty}\right]$, which we will henceforth do.

\section{ITERATing $\mathrm{PC}_{\mathcal{X}}^{\prime}$}

In this section we study what happens when we iterate $\mathrm{PC}_{\mathcal{X}}^{\prime}$, i.e., we look at $\mathrm{PC}_{\mathcal{X}}^{\prime} \star \mathrm{PC}_{\mathcal{X}}^{\prime}$. As mentioned in the introduction, we will show that $\mathrm{WWKL}^{\prime} \star$ $\mathrm{WWKL}^{\prime} \equiv_{W} \mathrm{PC}_{\omega \times 2^{\omega}}^{\prime}$. However, we will first show that $\mathrm{PC}_{\omega \times 2}^{\prime}$ is closed under iteration. For this, we relate $\mathrm{PC}_{\omega \times 2^{\omega}}^{\prime}$ to 2 -randomness. In what follows, we assume a fixed universal oracle Martin-Löf test $\mathcal{U}_{0}^{X}, \mathcal{U}_{1}^{X}, \ldots$

Definition 4.1. Let $X$ be $n$-random relative to $Y$. Then the $n$-randomness deficiency of $X$ relative to $Y$ is the least $m \in \omega$ such that $X \notin \mathcal{U}_{m}^{Y^{(n-1)}}$.

Definition 4.2. Let $\mathrm{WWKL}_{\neq 0 \omega}^{\prime}$ be the multi-valued function sending $\left(T_{i}\right)_{i \in \omega} \in$ limtree $2^{\infty}{ }^{\omega}$ to a non-zero element of $\left[T_{\infty}\right]$.

Similarly, let WWKL ${ }_{2-\text { Ran }}^{\prime}$ be the multi-valued function sending $\left(T_{i}\right)_{i \in \omega} \in \operatorname{limtree}_{2^{\omega}}^{>0}$ to an $X \in\left[T_{\infty}\right]$ which is 2 -random relative to $\left(T_{i}\right)_{i \in \omega}$.

Finally, let $\mathrm{WWKL}_{2-\mathrm{Ran}+\text { Def }}^{\prime}$ be the multi-valued function sending $\left(T_{i}\right)_{i \in \omega} \in$ limtree $2^{\infty}=0$ to an $X \in\left[T_{\infty}\right]$ which is 2-random relative to $\left(T_{i}\right)_{i \in \omega}$ and an upper bound on its 2-randomness deficiency relative to $\left(T_{i}\right)_{i \in \omega}$.

\section{Theorem 4.3.}

$$
\mathrm{PC}_{\omega \times 2^{\omega}}^{\prime} \equiv_{s W} \mathrm{WWKL}_{\neq 0^{\omega}}^{\prime} \equiv_{s W} \mathrm{WWKL}_{2-\text { Ran }}^{\prime} \equiv_{s W} \mathrm{WWKL}_{2-\text { Ran+Def }}^{\prime} .
$$

Proof. First, we show that $\mathrm{PC}_{\omega \times 2^{\omega}}^{\prime} \leq_{s W}$ WWKL ${ }_{\neq 0}^{\prime}$. Let $\left(T_{i}\right)_{i \in \omega} \in \operatorname{limtree}_{\omega \times 2^{\omega}}^{>0}$. Now let $\left(S_{i}\right)_{i \in \omega} \in$ limtree $_{2^{\omega}}^{>0}$ be the sequence of trees where

$$
S_{i}=\{\emptyset, 0,00, \ldots\} \cup \bigcup_{n \in \omega} 0^{n} 1 T_{i}^{n},
$$

where $T_{i}^{n}=\left\{\sigma \in 2^{<\omega} \mid n \sigma \in T_{i}\right\}$. Then

$$
\left[S_{\infty}\right]=\left\{0^{\omega}\right\} \cup \bigcup_{n \in \omega} 0^{n} 1\left[T_{\infty}^{n}\right],
$$

so the measure of $S_{\infty}$ is the same as the measure of $T_{\infty}$; in particular we see that indeed $\left(S_{i}\right)_{i \in \omega} \in$ limtree $_{2 \omega}^{>0}$. Furthermore, every $X \in\left[S_{\infty}\right]$ different from $0^{\omega}$ computes an element of $\left[T_{\infty}\right]$ by sending $0^{n} 1 Y$ to $n Y$.

Next, it is clear that every 2-random is different from $0^{\omega}$, which shows that $\mathrm{WWKL}_{\neq 0^{\omega}}^{\prime} \leq_{s W} \mathrm{WWKL}_{2-\mathrm{Ran}}^{\prime}$. We also get $\mathrm{WWKL}_{2-\mathrm{Ran}}^{\prime} \leq_{s W} \mathrm{WWKL}_{2-\mathrm{Ran}+\text { Def }}^{\prime}$ by just forgetting the bound on the randomness deficiency.

Finally, we show that $\mathrm{WWKL}_{2-\operatorname{Ran}+\text { Def }}^{\prime} \leq_{s W} \mathrm{PC}_{\omega \times 2^{\omega}}^{\prime}$. Given any $\left(T_{i}\right)_{i \in \omega} \in$ limtree $2^{\omega}$, we can uniformly compute trees $P_{i}^{n} \in \operatorname{Tree}_{2^{\omega}}^{>0}$ such that $\left(P_{i}^{n}\right)_{i \in \omega}$ converges to a tree $P_{\infty}^{n}$ with $\left[P_{\infty}^{n}\right]=2^{\omega} \backslash \mathcal{U}_{n}^{\left(T_{i}\right)_{i \in \omega}^{\prime}}$. Now, consider the sequence of trees $\left(S_{i}\right)_{i \in \omega}$ with $S_{i}$ given by:

$$
S_{i}=\bigcup_{n \in \omega} n\left(P_{i}^{n} \cap T_{i}\right)
$$

Then $\left(S_{i}\right)_{i \in \omega}$ is uniformly computable in $\left(T_{i}\right)_{i \in \omega}$. Let $n \in \omega$ and $q>0$ be such that $\mu_{2} \omega\left(T_{\infty}\right) \geq 2^{-n}+q$, which exists because $T_{\infty}$ has positive measure. Then 
$\mu_{2^{\omega}}\left(P_{\infty}^{n} \cap T_{\infty}\right) \geq q$, so also $\mu_{\omega \times 2^{\omega}}\left(S_{\infty}\right) \geq q>0$. Therefore $\left(S_{i}\right)_{i \in \omega} \in$ limtree ${ }_{\omega \times 2^{\omega}}^{>0}$. Finally, for every element of $n X \in\left[S_{\infty}\right]$ we have that $X \in\left[T_{\infty}\right]$, that $X$ is 2-random relative to $\left(T_{i}\right)_{i \in \omega}$ and that $n$ is a bound on its 2-randomness deficiency relative to $\left(T_{i}\right)_{i \in \omega}$.

To show that $\mathrm{PC}_{\omega \times 2^{\omega}}^{\prime}$ is closed under composition, we use the following lemma (see [1, Proposition 2.12] for a proof with $Y=\emptyset^{\prime}$, which relativizes in a straightforward way).

Lemma 4.4. There is a single Turing functional $\Phi$ such that for every 2-random $X$ relative to $Y$, if $n$ bounds the 2-randomness deficiency of $X$ relative to $Y$ then $\Phi\left(X \oplus Y^{\prime}, n\right)=(X \oplus Y)^{\prime}$.

Theorem 4.5. We have

$$
\mathrm{WWKL}_{2-\operatorname{Ran}}^{\prime} \star \mathrm{WWKL}_{2-\mathrm{Ran}+\operatorname{Def}}^{\prime} \equiv_{W} \mathrm{PC}_{\omega \times 2^{\omega}}^{\prime},
$$

and hence also by Theorem 4.3:

$$
\mathrm{PC}_{\omega \times 2^{\omega}}^{\prime} \star \mathrm{PC}_{\omega \times 2^{\omega}}^{\prime} \equiv_{W} \mathrm{PC}_{\omega \times 2^{\omega}}^{\prime}
$$

Proof. The fact that $\mathrm{WWKL}_{2-\mathrm{Ran}}^{\prime} \star \mathrm{WWKL}_{2-\mathrm{Ran}+\text { Def }}^{\prime} \geq_{W} \mathrm{PC}_{\omega \times 2^{\omega}}^{\prime}$ is a direct consequence of Theorem 4.3.

For the converse, let $f$ and $g$ be multi-valued functions such that both $f \leq_{W}$

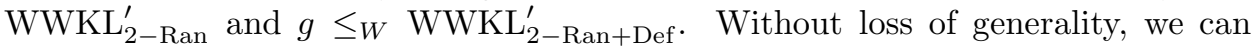
assume that the domain and range of $f$ and $g$ are contained in $\omega^{\omega}$. We want to show that $f \circ g \leq_{W} \mathrm{PC}_{\omega \times 2^{\omega}}^{\prime}$. Unfolding the definition of Weihrauch reducibility, and using the assumption on $f$ and $g$, we know that there exist three computable functions $T: \omega^{\omega} \rightarrow$ limtree $_{2 \omega}^{>0}, S: \subseteq \omega^{\omega} \times 2^{\omega} \times \omega \rightarrow$ limtree $_{2 \omega}^{>0}$ and $H: \subseteq \omega^{\omega} \times 2^{\omega} \times \omega \times 2^{\omega} \rightarrow \omega^{\omega}$ such that for every $X, Y, n$, such that $Y \in[\lim T(X)]$ and $Y$ is 2-random relative to $X$ with 2-randomness deficiency at most $n$, we have that $(X, Y, n)$ is in the domain of $S$, and for every $Z \in[\lim S(X, Y, n)], H(X, Y, n, Z) \in(f \circ g)(X)$.

The core of the argument is to show that for all $X$, the set $Q(X)$ of pairs $(n, Y \oplus Z)$ such that $Y \in[\lim T(X)], Y$ is 2-random relative to $X$ with 2-randomness deficiency at most $n$ and $Z \in \lim [S(X, Y, n)]$ is a $\Pi_{1}^{0}\left(X^{\prime}\right)$ subset of $\omega \times 2^{\omega}$, uniformly in $X$. This is a consequence of Lemma 4.4. Indeed, given $X$ the sequence $T(X)$ is computable in $X$, thus $\lim T(X)$ is $X^{\prime}$-computable, uniformly in $X$. Thus, the set of pairs $(Y, n)$ such that $Y$ is a path of $\lim T(X)$ and $Y$ is 2-random relative to $X$ with randomness deficiency at most $n$ is $\Pi_{1}^{0}\left(X^{\prime}\right)$ uniformly in $X$. Furthermore, the tree $\lim S(X, Y, n)$ is $(X, Y, n)^{\prime}$-computable uniformly, but because of Lemma 4.4 it is in fact $\left(X^{\prime}, Y, n\right)$-computable uniformly. Thus the set of paths of $\lim S(X, Y, n)$ is $\Pi_{1}^{0}\left(X^{\prime} \oplus Y\right)$. Putting all this together, we get that $Q(X)$ is indeed $\Pi_{1}^{0}\left(X^{\prime}\right)$ uniformly in $X$.

Furthermore, $Q(X)$ is a subset of $\omega \times 2^{\omega}$ of positive measure: given $X$, there is a positive probability that $Y$ chosen at random (w.r.t. the uniform measure) is in [lim $T(X)$ ] (because $T(X)$ has positive measure!), a positive probability that an integer chosen at random bounds the 2-randomness deficiency of $Y$ relative to $X$, and conditional to this, a positive probability that $Z$ chosen at random belongs to $[S(X, Y, n)]$ (which, assuming $Y$ and $n$ are as above, has positive measure).

We have established that $Q(X)$ is a $\Pi_{1}^{0}\left(X^{\prime}\right)$ subset of $\omega \times 2^{\omega}$ uniformly in $X$, hence can be represented by an $X^{\prime}$-computable tree over $\omega \times 2^{<\omega}$, and thus as the limit of an $X$-computable sequence of trees over $\omega \times 2^{<\omega}$. Now we immediately get the desired result: given $X$, one can compute a sequence in limtree ${ }_{\omega \times 2^{\omega}}^{>0}$ representing $Q(X)$, and for any path $(n, Y \oplus Z)$ of the limit tree (that is, a member of $Q(X))$, we get an element of $(f \circ g)(X)$ by simply computing $H(X, Y, n, Z)$. This shows $f \circ g \leq_{W} \mathrm{PC}_{\omega \times 2^{\omega}}^{\prime}$, as wanted. 
Thus, in particular we see that $\mathrm{WWKL}^{\prime} \star \mathrm{WWKL}^{\prime} \leq_{W} \mathrm{PC}_{\omega \times 2^{\omega}}^{\prime}$. Perhaps surprisingly, the converse is also true, which is expressed by the next theorem.

\section{Theorem 4.6.}

$$
\mathrm{WWKL}_{2-\mathrm{Ran}}^{\prime} \equiv_{W} \mathrm{WWKL}^{\prime} \star \mathrm{WWKL}^{\prime} .
$$

Proof. We need to show that $\mathrm{WWKL}_{2-\mathrm{Ran}}^{\prime} \leq_{W} \mathrm{WWKL}^{\prime} \star \mathrm{WWKL}^{\prime}$. Our idea is as follows. Given a $\left(T_{i}\right)_{i \in \omega} \in$ limtree $_{2 \omega}^{>0}$, we want to know the measure of $T_{\infty}$, so that we can intersect it with a large enough set of 2-randoms. Using the first instance of $\mathrm{WWKL}^{\prime}$, we will compute an $X$ such that $X^{\prime}$ computes a lower bound on the measure of $T_{\infty}$. To do this, our construction uses a partition $\left(I_{n}\right)_{n \in \omega}$ of $\omega$, where the $I_{n}$ should be sufficiently large. We build our first tree to which we apply WWKL' in such a way that $X \uparrow I_{n}$ is constantly 0 for $X$ on this tree if and only if the measure of $T_{\infty} \uparrow n$ (i.e., the measure of $\left\{x \in 2^{\omega} \mid x \uparrow n \in T_{\infty}\right\}$ ) drops significantly lower than the measure of $T_{\infty}\lceil(n-1)$. If we define 'significantly' in the right way, this will only happen finitely often; hence if our intervals $I_{n}$ are large enough we do not lose too much measure by adding this restriction. Furthermore, $X^{\prime}$ can compute how often $X \uparrow I_{n}$ is constantly 0 , and hence compute a lower bound on the measure of $T_{\infty}$.

We now give the details. Let $\left(T_{i}\right)_{i \in \omega} \in \operatorname{limtree}_{2 \omega}^{>0}$. Define a partition of $\omega$ by $I_{n}=[n(n+1),(n+1)(n+2))$; hence each $I_{n}$ has $2(n+1)$ elements. We define $\left(S_{i}\right)_{i \in \omega} \in$ limtree $2^{\omega}$ as follows. Each $S_{i}$ will be of the form $S_{i}^{0} S_{i}^{1} \ldots$, where each $S_{i}^{n} \subseteq\{0,1\}^{2(n+1)}$. In other words, a string $\sigma$ is in $S_{i}$ if and only if for each $n \in \omega$ we have $\sigma \uparrow I_{n} \in S_{i}^{n}$.

We show how to define $S_{i}^{n}$ by recursion on $n$. Simultaneously, we will define an auxiliary $k_{i, n} \in \omega$, where we initialise $k_{i,-1}=0$. Fix $i, n \in \omega$. We consider two cases:

- $\mu\left(T_{i}\lceil n)<2^{-k_{i, n-1}}\right.$. Then we let $S_{i}^{n}=\left\{0^{2(n+1)}\right\}$. Let $k_{i, n}$ be least such that $\mu\left(T_{i}\lceil n) \geq 2^{-k_{i, n}}\right.$ if $\mu\left(T_{i}\lceil n)>0\right.$; otherwise let $k_{i, n}=0$.

- $\mu\left(T_{i}\lceil n) \geq 2^{-k_{i, n-1}}\right.$. Then we let $S_{i}^{n}=\{0,1\}^{2(n+1)} \backslash\left\{0^{2(n+1)}\right\}$. Let $k_{i, n}=k_{i, n-1}$.

Then for every $n \in \omega$ we have that $\left(S_{i}^{n}\right)_{i \in \omega}$ converges to some tree $S_{\infty}^{n}$, because if $s$ is large enough such that $T_{i}$ has settled below $n$ for $i \geq s$, then $S_{i}^{n}=S_{j}^{n}$ for all $i, j \geq s$. For the same reason, $\left(k_{i, n}\right)_{i \in \omega}$ converges to some $k_{n}$. Since $T_{\infty}$ has positive measure, note that every $k_{n}$ is positive. In fact, $\left(k_{n}\right)_{n \in \omega}$ converges to the least $k \in \omega$ with $\mu\left(\left[T_{\infty}\right]\right) \geq 2^{-k}$.

We also claim that $S_{\infty}$ has positive measure. Let $m$ be large enough such that $k_{n}=k_{m}$ for all $n \geq m$. Then $S_{\infty}^{n}=\{0,1\}^{2(n+1)} \backslash\left\{0^{2(n+1)}\right\}$ for $n \geq m$. Fix any string $\sigma \in S_{\infty}^{0} S_{\infty}^{1} \ldots S_{\infty}^{m-1}$. Then

$$
\mu(\bar{S} \mid \sigma) \leq \sum_{n \geq m} 2^{-2(n+1)}<1,
$$

hence

$$
\mu(S) \geq 2^{-m(m+1)} \mu(S \mid \sigma)>0 .
$$

Now, given any $X \in\left[S_{\infty}\right]$, note that the number of $n$ such that $X \uparrow I_{n}=0^{2(n+1)}$ is exactly $k$. Thus, there is a Turing functional $\Phi$, independent of $\left(T_{i}\right)_{i \in \omega}$, such that for every $X \in\left[S_{\infty}\right]$ we have that $\Phi(X, i)$ converges to $k$ as $i$ goes to infinity.

Next, we define $\left(P_{i}\right)_{i \in \omega} \in$ limtree $_{2^{\omega}}^{>0}$ uniformly in $X$ and $\left(T_{i}\right)_{i \in \omega}$. Let $P_{i}=$ $T_{i} \cap \overline{\mathcal{U}_{\Phi(X, i)+1}^{\emptyset^{\prime}[i]}}$. Then $\left(P_{i}\right)_{i \in \omega}$ converges to $T_{\infty} \cap \overline{\mathcal{U}_{k+1}^{\emptyset^{\prime}}}$ which has positive measure and only has 2-random paths, as desired. 
It is known from Brattka and Pauly [7, Proposition 22] that $\mathrm{PC}_{\omega \times 2^{\omega}}>_{W}$ WWKL. Relativising this result we also get that $\mathrm{PC}_{\omega \times 2^{\omega}}^{\prime}>_{W} \mathrm{WWKL}$, and hence WWKL' $\mathrm{W}^{\prime}$ $\mathrm{WWKL}^{\prime}>_{W} \mathrm{WWKL}^{\prime}$.

Proposition 4.7. We have $\mathrm{WWKL}^{\prime} \leq_{s W} \mathrm{PC}_{\omega \times 2^{\omega}}^{\prime}$, but $\mathrm{PC}_{\omega \times 2^{\omega}}^{\prime} \underline{L}_{W} \mathrm{WWKL}$.

Proof. Clearly, WWKL' $\leq_{W} \mathrm{PC}_{\omega \times 2^{\omega}}^{\prime}$. For the converse, consider the multi-valued function $\mathrm{C}_{\omega}$ which assigns to a non-surjective function $f \in \omega^{\omega}$ an element not in the range of $f$; in other words, the computable instances represent finding an element of a non-empty co-c.e. set. ${ }^{1}$ Then $\mathrm{C}_{\omega}^{\prime} \leq_{s W} \mathrm{PC}_{\omega \times 2^{\omega}}^{\prime}$ : given $\left(f_{i}\right)_{i \in \omega}$, consider $\left(T_{i}\right)_{i \in \omega} \in$ limtree $_{\omega \times 2^{\omega}}^{>0}$ given by

$$
T_{i}=\bigcup_{j \in \omega} \bigcup_{n \notin f_{i}(\{0, \ldots, j\})} n 2^{<j} .
$$

Then $T_{i}$ is uniformly computable in $\left(T_{i}\right)_{i \in \omega}$, and it converges to

$$
T_{\infty}=\bigcup_{j \in \omega} \bigcup_{n \notin f_{\infty}(\{0, \ldots, j\})} n 2^{<j} .
$$

Furthermore, $T_{\infty}$ has positive measure because $f_{\infty}$ is not surjective. Thus, $\left(T_{i}\right)_{i \in \omega}$ is indeed an element of limtree $\underset{\omega \times 2}{>0}$. Finally, every element $X$ of $\left[T_{\infty}\right]$ computes an element not in the range of $f$, namely $X(0)$.

However, $\mathrm{C}_{\omega}^{\prime} \underline{Z}_{W}$ WWKL' (as pointed out by the referee, this also follows from [4, Corollary 12.3]; but we give a direct proof). Indeed, assume there are $K: \subseteq$ $\omega^{\omega} \rightarrow \operatorname{limtree}_{2}{ }^{\omega}$ and $H: \subseteq \omega^{\omega} \times 2^{\omega} \rightarrow \omega$ such that for every $f$ with $\left(f^{[i]}\right)_{i \in \omega}$ converging to some $f_{\infty}$ with $\operatorname{ran}\left(f_{\infty}\right) \neq \omega$ we have that $K(f)$ is total, and for every $X$ on $K(f)_{\infty}$ we have $H(f, X) \notin \operatorname{ran}\left(f_{\infty}\right)$. Given such an $f$ the complement of its range is a non-empty set $A$ which is co-c.e. in $\emptyset^{\prime}$, and for every non-empty set $A$ which is co-c.e. in $\emptyset^{\prime}$ we can effectively find an index for such a function $f$ from an index for $A$, so we we will implicitly identify these two. We will therefore build a set $A$ which is co-c.e. in $\emptyset^{\prime}$ for which $H(f, X) \notin A$ for any $X \in K(f)_{\infty}$.

By the recursion theorem we may assume we know an index $e$ for $f$. So, using $\emptyset^{\prime}$ and $e$ we can compute $K(\{e\})_{\infty}$. Now look for the least $s$ such that for every $\sigma \in$ $K(\{e\})_{\infty}$ of length $s$ we have that $H(\{e\}, \sigma)[s] \downarrow$ if such an $s$ exists, and enumerate the finitely many values $H(\{e\}, \sigma)$ into the complement of $A$. If such an $s$ does not exist, we let $A=\omega$.

Now we know by compactness that an $s$ as above exists, and therefore $A$ is cofinite (hence non-empty). However, by construction we now have that $U(\{e\}, X) \notin$ $A$ for every $X$ on $K(\{e\})_{\infty}$, which is a contradiction.

Next, we separate $\mathrm{WWKL}_{2-\text { Ran }}^{\prime}$ and hence $\mathrm{WWKL}^{\prime} \star \mathrm{WWKL}^{\prime}$ from $\mathrm{WWKL}^{\prime \prime}$, using randomness and the effective 0-1-law. It is known from Brattka and Pauly [7] that $\mathrm{PC}_{\omega \times 2^{\omega}}<_{W} \mathrm{WWKL}^{\prime}$. We now show that this also holds one jump higher.

Proposition 4.8. We have $\mathrm{WWKL}_{2-\mathrm{Ran}} \leq_{s W} \mathrm{WWKL}^{\prime \prime}$, but on the other hand $\mathrm{WWKL}^{\prime \prime} \not_{W} \mathrm{WWKL}_{2-\mathrm{Ran}}^{\prime}$.

Proof. Given any $\left(T_{i}\right)_{i \in \omega} \in \operatorname{limtree}_{2^{\omega}}^{>0}$, we can use $\left(T_{i}\right)_{i \in \omega}^{\prime \prime}$ to compute a lower bound $2^{-n}$ on the measure of $T_{\infty}$, and then let $S$ be a tree such that $[S]=\left[T_{\infty}\right] \backslash$ $\mathcal{U}_{n+1}^{\left(T_{i}\right)_{i \in \omega}^{\prime}}$. Since $S$ is $\left(T_{i}\right)_{i \in \omega^{\prime}}^{\prime \prime}$-computable we can uniformly transform $S$ into a $\left(T_{i}\right)_{i \in \omega^{-}}$ computable double sequence $\left(\left(S_{i}^{j}\right)_{i \in \omega}\right)_{j \in \omega}$ with $\lim _{j \rightarrow \infty} \lim _{i \rightarrow \infty} S_{i}^{j}=S$. Since every infinite path of $S$ is a 2-random ${ }^{\left(T_{i}\right)_{i \in \omega}}$ path of $T_{\infty}$ and all the computations are uniform, this shows that $\mathrm{PC}_{\omega \times 2^{\omega}}^{\prime} \leq_{s W}$ WWKL".

\footnotetext{
${ }^{1}$ Our definition is not exactly the same as the definition of $\mathrm{C}_{\omega}$ in Brattka, Gherardi and Hölzl [3], but it is easily seen to be strongly Weihrauch-equivalent to it.
} 
Conversely, assume towards a contradiction that WWKL" $\leq_{W} \mathrm{WWKL}_{2-\mathrm{Ran}^{\prime}}$. Consider any $\emptyset^{\prime \prime}$-computable tree $S$ of positive measure without $\emptyset^{\prime \prime}$-computable paths. Then $S$ is the limit of a computable double sequence $\left(\left(S_{i}^{j}\right)_{i \in \omega}\right)_{j \in \omega}$ converging to $S$. Therefore, from our assumption that WWKL" $\leq_{W} \mathrm{WWKL}_{2-\text { Ran }}^{\prime}$ it follows that there should be a computable $\left(T_{i}\right)_{i \in \omega} \in \operatorname{limtree}_{2 \omega}^{>0}$ such that every 2-random path of $T_{\infty}$ computes a path of $S$. Let $X$ be a $\emptyset^{\prime \prime}$-computable 2 -random set. Then the effective 0-1-law tells us that $Y=\sigma X$ is a path of $T_{\infty}$ for some $\sigma \in 2^{<\omega}$. So, $Y$ is a 2 -random path of $T_{\infty}$, but since it is $\emptyset^{\prime \prime}$-computable it clearly does not compute a path of $S$.

Thus, combining everything from this section we have the following.

Corollary 4.9.

$$
\mathrm{WWKL}^{\prime}<_{W} \mathrm{WWKL}^{\prime} \star \mathrm{WWKL}^{\prime}<_{W} \mathrm{WWKL}^{\prime \prime} .
$$

5. $\mathrm{PC}_{\omega^{\omega}}$

In this section we will show that $\mathrm{PC}_{\omega^{\omega}} \equiv_{s W} \mathrm{PC}_{\omega \times 2^{\omega}}$. First, let us remark that, replacing every occurrence of 2-randomness by 1-randomness in Definition 4.2 and Theorem 4.3, we also get the following result by the same proof as for Theorem 4.3.

\section{Theorem 5.1.}

$$
\mathrm{PC}_{\omega \times 2^{\omega}} \equiv_{s W} \mathrm{WWKL}_{\neq 0} \equiv_{s W} \mathrm{WWKL}_{1-\mathrm{Ran}} \equiv_{s W} \mathrm{WWKL}_{1-\text { Ran+Def }} .
$$

The following result, due to Brattka, Gherardi and Hölzl, was previously announced by Hölzl at ARA 2014 in Gotemba, but a proof has not yet appeared in print.

Theorem 5.2.

$$
\mathrm{PC}_{\omega} \omega \equiv_{s W} \mathrm{PC}_{\omega \times 2^{\omega}} \text {. }
$$

Proof. Clearly $\mathrm{PC}_{\omega \times 2} \leq_{s W} \mathrm{PC}_{\omega^{\omega}}$. We show the converse. Using the previous theorem, this is equivalent to showing that $\mathrm{PC}_{\omega^{\omega}} \leq_{s W} \mathrm{WWKL}_{1-\mathrm{Ran}}$.

We will use the function $\alpha: 2^{<\omega} \rightarrow \omega^{<\omega}$ which maps a string $\sigma$ to the string enumerating $\sigma$ in increasing order, i.e., the length of $\alpha(\sigma)$ is the number of ones in $\sigma$, and $\alpha(\sigma)(n)$ is the position of the $n$th one.

First we define a computable function $K$ which maps trees in $\omega^{\omega}$ to trees in $2^{\omega}$. Let $T$ be a tree in $\omega^{\omega}$. Given any string $\sigma \in 2^{<\omega}$, we put $\sigma$ into $K(T)$ if and only if $\alpha(\sigma) \in T$.

Then $K$ is clearly computable. Furthermore, if $X \in[K(T)]$ has infinitely many ones, then $\alpha(X):=\bigcup_{n \in \omega} \alpha(X \uparrow n) \in \omega^{\omega}$ is an infinite path of $T$. In particular this holds for the random paths of $K(T)$, thus every random path of $K(T)$ computes a path of $T$. Finally, by the way we have defined our measures we know that $\alpha$ is measure-preserving, and hence

$$
\mu_{2^{\omega}}([K(T)]) \geq \mu_{2^{\omega}}\left(\alpha^{-1}([T])\right)=\mu_{\omega^{\omega}}([T])>0 .
$$

Note that this proof directly relativises, giving us the following result as well. Alternatively, this follows from the fact that if $f \leq_{s W} g$ then $f^{\prime} \leq_{s W} g^{\prime}$, as proven in Brattka, Gherardi and Marcone [4].

\section{Corollary 5.3.}

$$
\mathrm{PC}_{\omega^{\omega}}^{\prime} \equiv_{s W} \mathrm{PC}_{\omega \times 2^{\omega}}^{\prime}
$$




\section{MiXING JUMPS AND ITERATIONS}

Next, we wonder: what happens if we mix the composition of WWKL and WWKL'? First, let us show that WWKL $\star \mathrm{WWKL}^{\prime} \equiv_{W} \mathrm{WWKL}^{\prime}$.

\section{Proposition 6.1.}

\section{$\mathrm{WWKL} \star \mathrm{WWKL}^{\prime} \equiv_{W} \mathrm{WWKL}^{\prime}$.}

Proof. Let $H: \subseteq \operatorname{limtree}_{2^{\omega}}^{>0} \times 2^{\omega} \rightarrow$ Tree $_{2^{\omega}}^{>0}$ be a Turing functional. We show how to, given $\left(T_{i}\right)_{i \in \omega} \in \operatorname{limtree} 2^{>\omega}$ such that $\left(\left(T_{i}\right)_{i \in \omega}, X\right) \in \operatorname{dom}(H)$ for every $X \in\left[T_{\infty}\right]$, uniformly construct an $\left(S_{i}\right)_{i \in \omega} \in$ limtree $_{2 \omega}^{>0}$ such that every path $X$ of $S_{\infty}$ uniformly computes a path of both $T_{\infty}$ and $H\left(\left(T_{i}\right)_{i \in \omega}, X\right)$.

For this, we let $S_{i}$ be the tree where a string $\sigma \oplus \tau \in S_{i}$ if and only if $\sigma \in S_{i}$ and, if $H\left(\left(T_{i}\right)_{i \in \omega}, \sigma\right)[\sigma](\tau) \downarrow$, then $H\left(\left(T_{i}\right)_{i \in \omega}, \sigma\right)(\tau)=1$. Then it is not hard to verify that $\left(S_{i}\right)_{i \in \omega}$ converges to some tree $S_{\infty}$. Furthermore, for every $X \in\left[T_{\infty}\right]$ and $Y \in\left[H\left(\left(T_{i}\right)_{i \in \omega}, X\right)\right]$ we have that $X \oplus Y \in\left[S_{\infty}\right]$, and vice versa. Finally, by Fubini's theorem, $S_{\infty}$ has positive measure, completing the proof.

It turns out the converse also holds. In fact, we even have that WWKL' * $\mathrm{PC}_{\omega \times 2} \equiv_{W} \mathrm{WWKL}^{\prime}$.

\section{Theorem 6.2.}

$$
\mathrm{WWKL}^{\prime} \star \mathrm{PC}_{\omega \times 2^{\omega}} \equiv_{W} \mathrm{WWKL}^{\prime} \star \mathrm{WWKL} \equiv_{W} \mathrm{WWKL}^{\prime} .
$$

Proof. Clearly,

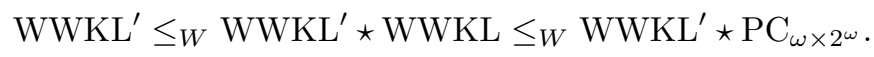

We need to show that $\mathrm{WWKL}^{\prime} \star \mathrm{PC}_{\omega \times 2^{\omega}} \leq_{W} \mathrm{WWKL}^{\prime}$; or, by Theorem 5.1 , that $\mathrm{WWKL}^{\prime} \star \mathrm{WWKL}_{1-\mathrm{Ran}+\text { Def }} \leq_{W} \mathrm{WWKL}^{\prime}$. In fact, we show that even

$$
\mathrm{WWKL}^{\prime} \star \mathrm{WWKL}_{2-\mathrm{Ran}+\mathrm{Def}} \leq_{W} \mathrm{WWKL}^{\prime}
$$

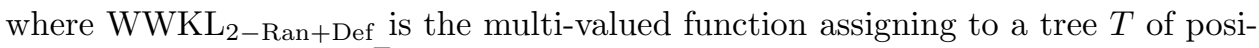
tive measure a 2 -random ${ }^{T}$ path through $T$ together with a bound on its 2 -randomness ${ }^{T}$ deficiency. The proof is a variation on the proof of Theorem 4.5. Let $f$ and $g$ be multi-valued functions such that $f \leq_{W} \mathrm{WWKL}^{\prime}$ and $g \leq_{W} \mathrm{WWKL}_{2-\mathrm{Ran}+\text { Def. }}$. Again, without loss of generality, we assume that the domain and range of $f$ and $g$ are contained in $\omega^{\omega}$. We want to show that $f \circ g \leq_{W} \mathrm{WWKL}^{\prime}$. This time, we have three computable functions $T: \omega^{\omega} \rightarrow$ Tree $_{2 \omega}^{>0}, S: \subseteq \omega^{\omega} \times 2^{\omega} \times \omega \rightarrow$ limtree $_{2 \omega}^{>0}$ and $H: \subseteq \omega^{\omega} \times 2^{\omega} \times \omega \times 2^{\omega} \rightarrow \omega^{\omega}$ such that for every $X, Y, n$, such that $Y \in[T(X)]$ and $Y$ is 2-random relative to $X$ with 2-randomness deficiency at most $n$, we have that $(X, Y, n)$ is in the domain of $S$, and for every $Z \in[\lim S(X, Y, n)]$, $H(X, Y, n, Z) \in(f \circ g)(X)$.

Given $X$, we can compute, uniformly relative to $X^{\prime}$, an $n=n(X)$ such that $[T(X)]$ has measure strictly greater than $2^{-n}$ and thus there is some member of $T[X]$ of 2-randomness deficiency at most $n$. Similarly to the proof of Theorem 4.5 , the set $Q(X)$ of sequences $Y \oplus Z$ such that $Y$ belongs to $T(X)$ and is 2-random relative to $X$ with 2-randomness deficiency bounded by $n(X)$, and $Z \in[\lim S(X, Y, n)]$ is a $\Pi_{1}^{0}\left(X^{\prime}\right)$ subset of $2^{\omega}$, uniformly in $X^{\prime}$ (and has positive measure). Thus one can, given $X$, uniformly compute a sequence of trees converging to a tree whose paths are members of $Q(X)$, and for every such path $Y \oplus Z$, one gets a member of $(f \circ g)(X)$ by computing $H(X, Y, n(X), Z)$.

Thus, we have now studied all combinations of WWKL, WWKL', $\mathrm{PC}_{\omega \times 2^{\omega}}$ and $\mathrm{PC}_{\omega \times 2^{\omega}}^{\prime}$, except for $\mathrm{PC}_{\omega \times 2^{\omega}} \star \mathrm{WWKL}^{\prime}$. An earlier draft of this paper contained an incorrect statement about this principle, which was pointed out by Brattka and Hölzl. In fact, they pointed out the following could be proven using techniques from this paper. 
Lemma 6.3. (Brattka and Hölzl [private communication]) Let Fin $\subseteq 2^{\omega}$ be the set of binary sequences with only finitely many ones. Then

$$
\operatorname{Id}_{\text {Fin }} \leq_{s W} \text { WWKL. }
$$

Proof. As in the proof of Theorem 4.6, let $I_{n}=[n(n+1),(n+1)(n+2))$. Given any $X \in$ Fin, let $T$ be the tree such that $Y \in[T]$ if and only if, for all $n \in \omega$ we have that $Y \uparrow I_{n}=0^{2(n+1)}$ if and only if $X(n)=1$. Then $T$ has positive measure because $X$ only contains finitely many ones, as in the proof of Theorem 4.6. Furthermore, any path $Y$ through $T$ clearly computes $X$, as desired.

Lemma 6.4. (Brattka and Hölzl [private communication])

$$
\mathrm{C}_{\omega}^{\prime} \leq_{W} \mathrm{C}_{\omega} \star \mathrm{WWKL}^{\prime}
$$

Proof. From the previous lemma, together with the fact that, if $f \leq_{s W} g$, then $f^{\prime} \leq_{s W} g^{\prime}$ (Brattka, Gherardi and Marcone [4]), we see that $\operatorname{Id}_{\text {Fin }}^{\prime} \leq_{s W}$ WWKL'. Furthermore, from [4] we also know that $\operatorname{Id}_{\omega}^{\prime} \equiv_{s W} \mathrm{C}_{\omega}$. Thus, it is enough if we show that $\mathrm{C}_{\omega}^{\prime} \leq_{W} \operatorname{Id}_{\omega}^{\prime} \star \operatorname{Id}_{\text {Fin }}^{\prime}$.

Now, given any function $f$ converging to a non-surjective function, let $X$ be such that $X(\langle i, n, m\rangle)=1$ if and only if the least element not in the range of $f^{[i]}\lceil n+1$ is different from the least element not in the range of $f^{[i]} \uparrow n$, and this new least element is equal to $m$. Then $X$ converges to an element $X_{\infty} \in \mathrm{Fin}$, so the function mapping $f$ to $X_{\infty}$ is reducible to $\operatorname{Id}_{\text {Fin }}^{\prime}$.

Finally, given $X_{\infty}$, let $m$ be the largest element of the finite set $\{m \mid \exists n .\langle n, m\rangle \in$ $\left.X_{\infty}\right\}$. Since this $m$ can be found as the limit of an $X_{\infty}$-computable sequence, we obtain that $\mathrm{C}_{\omega}^{\prime} \leq_{W} \mathrm{C}_{\omega} \star \mathrm{WWKL}^{\prime}$, as desired.

Proposition 6.5. (Brattka and Hölzl [private communication])

$$
\mathrm{PC}_{\omega \times 2^{\omega}} \star \mathrm{WWKL}^{\prime} \equiv_{W} \mathrm{PC}_{\omega \times 2^{\omega}}^{\prime}
$$

Proof. That $\mathrm{PC}_{\omega \times 2^{\omega}} \star \mathrm{WWKL}^{\prime} \leq_{W} \mathrm{PC}_{\omega \times 2^{\omega}}^{\prime}$ follows directly from Theorem 4.5. For the converse, we use the previous lemma, together with the fact that, if $f \leq_{s W} g$, then $f^{\prime} \leq_{s W} g^{\prime}$, the fact that $\mathrm{PC}_{\omega \times 2} \omega \equiv_{s W} \mathrm{C}_{\omega} \times$ WWKL (Brattka, Gherardi and Hölzl $\left.[3]^{2}\right)$ and the easy fact that $\mathrm{WWKL}^{\prime} \equiv_{s W} \mathrm{WWKL}^{\prime} \times \mathrm{WWKL}^{\prime}$. We now have

$$
\begin{aligned}
& \mathrm{PC}_{\omega \times 2^{\omega}} \star \mathrm{WWKL}^{\prime} \equiv_{W}\left(\mathrm{C}_{\omega} \times \mathrm{WWKL}\right) \star \mathrm{WWKL}^{\prime} \\
& \geq_{W} \mathrm{C}_{\omega} \star \mathrm{WWKL}^{\prime} \\
& \equiv_{W}\left(\mathrm{C}_{\omega} \star \mathrm{WWKL}^{\prime}\right) \times \mathrm{WWKL}^{\prime} \\
& \geq_{W} \mathrm{C}_{\omega}^{\prime} \times \mathrm{WWKL}^{\prime} \\
& \equiv_{W}\left(\mathrm{C}_{\omega} \times \mathrm{WWKL}\right)^{\prime} \\
& \equiv_{W} \mathrm{PC}_{\omega \times 2^{\omega}}^{\prime} \text {. }
\end{aligned}
$$

We would like to finish this paper with an alternative proof of the separation of $W_{W K L}^{\prime} \star W W K L^{\prime}$ and WWKL'. The techniques for this proof were originally developed by the authors to separate $\mathrm{WWKL}^{\prime} \star \mathrm{WWKL}^{\prime}$ from $\mathrm{WWKL}^{\prime}$ before they knew that $\mathrm{WWKL}^{\prime} \star \mathrm{WWKL}^{\prime} \equiv_{W} \mathrm{PC}_{\omega \times 2^{\omega}}^{\prime}$. We hope this alternative approach, avoiding notions from algorithmic randomness, might be useful for other purposes in the future.

For this result, we will make use of another well-known Weihrauch degree, namely the degree LPO, which is the degree associated to the function (which we also denote by LPO for simplicity) sending $X \in 2^{\omega}$ to 0 if $X=0^{\omega}$ and to 1 otherwise.

The following was proven in [3]

\footnotetext{
${ }^{2}$ They do not explicitly state the strong Weihrauch equivalence, but it follows directly from their proof.
} 
Proposition 6.6. $\mathrm{LPO} \leq_{W} \mathrm{PC}_{\omega \times 2^{\omega}}$.

(Indeed, given $X$, one can compute the tree $T^{X}$ - of positive measure - such that $0 \sigma \in T^{X}$ if and only if $X \uparrow|\sigma|=0^{|\sigma|}$ and for $n>0, n \sigma \in T^{X}$ if and only if $X\left\lceil n\right.$ contains a 1 . Then, given a path $n Z$ of $T^{X}$, we have $n=0$ if and only if $\operatorname{LPO}(X)=0)$. This immediately gives us the following corollary.

\section{Corollary 6.7.}

\section{$\mathrm{LPO} \star \mathrm{WWKL}^{\prime} \leq_{W} \mathrm{WWKL}^{\prime} \star \mathrm{WWKL}^{\prime}$.}

However, we show that one cannot do this with just one application of WWKL'.

\section{Theorem 6.8.}

\section{$\mathrm{LPO} \star \mathrm{WWKL}^{\prime} \not_{W} \mathrm{WWKL}$}

Proof. Suppose for the sake of contradiction that LPO $\star \mathrm{WWKL}^{\prime} \leq_{W} \mathrm{WWKL}^{\prime}$. This means in particular that there exist two computable functions $K: \operatorname{limtree}_{2 \omega}^{>0} \times$ $\omega \rightarrow$ limtree $_{\omega \times 2^{\omega}}^{>0}$ and $H:$ limtree $_{2^{\omega}}^{>0} \times 2^{\omega} \rightarrow 2^{\omega} \times\{0,1\}$ such that for every $\left(T_{i}\right)_{i \in \omega} \in$ limtree $2_{2 \omega}^{>0}$, we have that for every path $X$ of $\lim K\left(\left(T_{i}\right)_{i \in \omega}\right)$ that $H\left(\left(T_{i}\right)_{i \in \omega}, X\right)=$ $(Z, \operatorname{LPO}(Z))$ for some path $Z$ of $T_{\infty}$. To get our contradiction, we will make use of the recursion theorem relative to $\emptyset^{\prime}$ to build a suitable $\left(T_{i}\right)_{i \in \omega}$. What we do is build a $\emptyset^{\prime}$-c.e. set of strings (elements of $2^{<\omega}$ ) $W_{e}^{\emptyset^{\prime}}$ whose index we know in advance. Then we get a $\emptyset^{\prime}$-computable tree $T$ by putting $\sigma \in T$ if and only if $\sigma$ has no prefix in $W_{e}^{\emptyset^{\prime}}[|\sigma|]$. We thus know a $\emptyset^{\prime}$-index for $T$, and therefore we also know an index for a computable sequence $\left(T_{i}\right)_{i \in \omega}$ of trees converging to $T$.

Now, we can $\emptyset^{\prime}$-compute $S=\lim K\left(\left(T_{i}\right)_{i \in \omega}\right)$ (to make sure this limit exists, we will need to ensure that $T$ has indeed positive measure, but we will see at the end of construction that it is indeed the case). Let $H_{0}$ and $H_{1}$ be the first and second projection of $H$, respectively. By compactness one can, relatively to $\emptyset^{\prime}$, find a clopen set $D$ such that $H_{1}\left(\left(T_{i}\right)_{i \in \omega}, X\right)=0$ for all $X \in\left[S_{0}\right]=[S] \cap D$ and $H_{1}\left(\left(T_{i}\right)_{i \in \omega}, X\right)=1$ for all $X \in\left[S_{1}\right]=[S] \cap D^{c}$. It is well-known that the image of an effectively compact set by a computable function which is total on this set is itself effectively compact (and an index of the image can be uniformly obtained from an index of the source). Relativizing this to $\emptyset^{\prime}$, we see that the image of $S_{1}$ under $X \mapsto H_{0}\left(\left(T_{i}\right)_{i \in \omega}, X\right)$ is a $\emptyset^{\prime}$-effectively compact set. This image cannot contain $0^{\omega}$ by definition of $\left[S_{1}\right]$ (because otherwise $H\left(\left(T_{i}\right)_{i \in \omega}, X\right)=\left(0^{\omega}, 1\right)$ for some $X \in[S]$, contradicting the assumption on $H$ and the definition of LPO). Therefore, we can $\emptyset^{\prime}$-effectively wait until we find an $l$ such that the image of $\left[S_{1}\right]$ under $X \mapsto H_{0}\left(\left(T_{i}\right)_{i \in \omega}, X\right)$ is disjoint from $\left[0^{l}\right]$. When such an $l$ is found, we enumerate in $W_{e}^{\emptyset^{\prime}}$ all strings that are incompatible with $0^{l} 1$, so as to get $[T]=\llbracket 0^{l} 1 \rrbracket$. Now, for any $X \in[S]$, either $X \in\left[S_{0}\right]$, in which case $H\left(\left(T_{i}\right)_{i \in \omega}, X\right)=(Z, 0)$ for some $Z \in[T]$, which is not possible because $\operatorname{LPO}(Z)=1$ for all $Z \in[T]$, or $X \in\left[S_{1}\right]$, in which case $H\left(\left(T_{i}\right)_{i \in \omega}, X\right)=(Z, 1)$ where $0^{l}$ is not a prefix of $Z$, and again this is not possible since $Z$ is supposedly in $[T]$. Noting that the tree $T$ does have positive measure as promised, we have obtained a contradiction.

Alternatively, the proof of the previous Theorem also follows from the fact that $\mathrm{LPO}^{\prime} \leq_{W} \mathrm{LPO} \star \mathrm{WWKL}^{\prime}$, using similar arguments as in Proposition 6.5, and the fact that $\mathrm{LPO}^{\prime} \mathbb{Z}_{W} \mathrm{WWKL}^{\prime}$ from [4].

\section{Corollary 6.9.}

$$
\mathrm{WWKL}^{\prime}<_{W} \mathrm{WWKL}^{\prime} \star \mathrm{WWKL}^{\prime} .
$$




\begin{tabular}{|c|c|c|c|c|}
\hline$\downarrow \star \rightarrow$ & WWKL & $\mathrm{PC}_{\omega \times 2^{\omega}}$ & WWKL' & $\mathrm{PC}_{\omega \times 2^{\omega}}^{\prime}$ \\
\hline $\begin{array}{c}\mathrm{WWKL} \\
\mathrm{PC}_{\omega \times 2^{\omega}} \equiv_{s W} \mathrm{PC}_{\omega^{\omega}} \\
\mathrm{WWKL}^{\prime} \\
\mathrm{PC}_{\omega \times 2^{\omega}}^{\prime} \equiv_{s W} \mathrm{PC}_{\omega^{\omega}}^{\prime}\end{array}$ & $\begin{array}{l}\text { WWKL } \\
\mathrm{PC}_{\omega \times 2 \omega} \\
\mathrm{WWKL}^{\prime} \\
\mathrm{PC}_{\omega \times 2^{\omega}}^{\prime}\end{array}$ & $\begin{array}{l}\mathrm{PC}_{\omega \times 2^{\omega}} \\
\mathrm{PC}_{\omega \times 2^{\omega}} \\
\mathrm{WWKL}^{\prime} \\
\mathrm{PC}_{\omega \times 2^{\omega}}^{\prime}\end{array}$ & $\begin{array}{l}\mathrm{WWKL}^{\prime} \\
\mathrm{PC}_{\omega \times 2^{\omega}}^{\prime} \\
\mathrm{PC}_{\omega \times 2 \omega}^{\prime} \\
\mathrm{PC}_{\omega \times 2^{\omega}}^{\prime}\end{array}$ & $\begin{array}{l}\mathrm{PC}_{\omega \times 2^{\omega}}^{\prime} \\
\mathrm{PC}_{\omega \times 2^{\omega}}^{\prime} \\
\mathrm{PC}_{\omega \times 2^{\omega}}^{\prime} \\
\mathrm{PC}_{\omega \times 2 \omega}^{\prime}\end{array}$ \\
\hline
\end{tabular}

\section{Summary}

The following table summarises our results.

Between these principles, we have the following relations:

$$
\mathrm{WWKL}<_{W} \mathrm{PC}_{\omega \times 2^{\omega}}<_{W} \mathrm{WWKL}^{\prime}<_{W} \mathrm{PC}_{\omega \times 2^{\omega}} \star \mathrm{WWKL}^{\prime}<_{W} \mathrm{PC}_{\omega \times 2^{\omega}}^{\prime} .
$$

Finally, let us remark that many of the results studied in this paper also hold for more than one jump, but to avoid cluttering the notation we have not mentioned these results explicitly.

\section{REFERENCES}

(Laurent Bienvenu) LIRMM, CNRS \& Université De Montpellier, 161 Rue AdA, 34095 Montpellier Cedex 5

E-mail address: laurent.bienvenu@computability.fr

(Rutger Kuyper) Department of Mathematics, University of Wisconsin-Madison, MadiSON, WI 53706, USA

E-mail address: mail@rutgerkuyper.com 